\title{
EVALUACIÓN DE UN EQUIPO PARA LA OBTENCIÓN MECÁNICA DE FIBRA DE RASTROJOS DE LA COSECHA DE PIÑA GOLDEN
}

\author{
EVALUATION OF AN EQUIPMENT FOR THE MECHANICAL OBTAINING OF TRACE
} FIBER FROM THE GOLDEN PINEAPPLE HARVEST

Percy Fermín Velásquez Cosi ${ }^{1}$ Víctor Berrocal Ventura ${ }^{2}$ John Joseph Cárdenas Canchari ${ }^{3}$

Información del artículo: Recibido: 01/03/2020 Aceptado: 20/11/2020

${ }^{1}$ Docente Escuela de Ingeniería Agroindustrial, Universidad Nacional San Cristóbal de Huamanga, Perú

${ }^{2,3}{ }^{3}$ Escuela de Ingeniería Agroindustrial, Universidad Nacional San Cristóbal de Huamanga, Perú E-mail: 1percy.velasquez@unsch.edu.pe, 2victorberrocalv26@gmail.com, 33zekaru_43@hotmail.com 


\section{Resumen}

La investigación tiene la finalidad de diseñar, construir y evaluar una máquina que tenga la capacidad de liberar la celulosa del resto de biomasa residual y así proporcionar un valor agregado a la fibra de la corteza de la piña. Se realizó un estudio teórico sobre la materia prima y la tecnología que implica la obtención de la fibra celulósica, donde es material potencial para la industria textil, curtiembre, pulpa de papel, etc. Por lo que, se procedió a diseñar los componentes del prototipo, por lo que resulta necesaria analizar y determinar los esfuerzos a los que están sometidos los mismos cuando el sistema mecánico (en el cual están integrados) se encuentra bajo unas determinadas condiciones de funcionamiento. La característica del diseño, es que posee (2) cuerpos muy diferenciados: móvil y estática, es preciso en la carga de la materia prima y es netamente para trabajos del tipo batch y especialmente es desarmable en sus componentes para mantenimiento.

Palabras clave: Diseño; desfibrador; celulosa; biomasa.

\section{Abstract}

The research aims to design, build and evaluate a machine that has the ability to release cellulose from the rest of residual biomass and thus provide added value to the pineapple bark fiber. A theoretical study was carried out on the raw material and technology involved in obtaining cellulosic fiber, where it is potential material for the textile industry, tanning, paper pulp, etc. Therefore, the components of the prototype were designed, so it is necessary to analyze and determine the efforts to which they are subjected when the mechanical system (in which they are integrated) is under certain operating conditions. The characteristic of the design is that it has (2) very differentiated bodies: mobile and static, it is accurate in the load of the raw material and is clearly for batch type jobs and especially is removable in its components for maintenance.

Keywords: Design; shredder; cellulose; biomass. 


\section{Introducción}

El promedio de generación de rastrojo por hectárea de piña es del 7 \% de acuerdo a Avella (2018), además la ausencia de estudios basados en el uso distinto de los residuos agrícolas consistentes en las cortezas foliares de la piña, que solo tiene un enfoque naturalmente de abono, sin embargo este proceso es lento por las características físicas y químicas de la planta, por lo cual se debe utilizar agentes químicos que contribuyan a su descomposición, Una de las problemáticas que enfrentan los productores de piña es poder realizar un manejo adecuado de los residuos que produce dicho cultivo posterior a su cosecha.

Actualmente en las localidades de San Gabán (Puno), Tingo María (Huánuco), Aguaytía (Ucayali), Puerto Bermúdez (Cerro de Pasco), VRAEM (Ayacucho- Cusco- Junín) y entre otros; existen proyectos de desarrollo del cultivo de piña en las variedades Cayena Lisa y Golden, promovidas por los Gobiernos Regionales, las Municipalidades y Organismos No Gubernamentales (ONG) que hacen un total de 600 a 800 hectáreas de nuevas áreas y [comercialmente] todas para entrar al mercado de Lima; sin embargo, las localidades con las mayores áreas plantadas de piña siguen siendo Chanchamayo y Satipo, donde la tecnología usada es relativamente mejor a otras zonas del país (MINAGRI, 2018). La fibra vegetal de hoja de piña es una alternativa realmente llamativa para la agroindustria por ser una material renovable y biodegradable, se utiliza como refuerzo en materiales compuesto de matriz polimérica. Su uso se da más en el sector no alimentario; como material polímero como refuerzo de fibras para la elaboración de calzados, sombreros vestidos, alfombras, sogas, costales, papel, gasas, mallas de invernaderos, de sombras, redes de pesca (debido a su resistencia al agua salada).

En el sector alimentario, sirve de bioenvoltorios, bolsos de papel, envolturas de embutidos, fundas de té, platos biodegradables, ya que está entrando con gran demanda el uso de envoltorios biodegradables a causa de la feroz contaminación mundial, todo concerniente a la protección o recubrimiento de algún alimento.

Actualmente por los problemas y efectos del cambio climático, están obligando que tengamos mejores alternativas de productos ecoamigables con el medio ambiente y que genere adicionalmente una dinámica económica entorno del productor; por lo que, es importante la valorización de estos residuos mediante la producción de fibras celulósicas seleccionados por sus óptimas propiedades fisicoquímicas y su relevancia en el sector agroindustrial peruano. Entonces, para aprovechar esta materia prima bruta, en diversos productos agroindustriales sostenibles y sustentables con el medio ambiente, como una alternativa a la industria textil, de curtiembre y de pulpa de papel, es el desarrollo del prototipo en investigación.

\section{Objetivos}

Evaluar el prototipo desfibrador, para la obtención de fibra natural, a partir de los residuos foliares de la piña, en la región de Ayacucho.

\section{Metodología}

El trabajo de investigación se desarrolló en la empresa COVE Ingenieros SAC y el Centro Experimental de Curtiembre de la Facultad de Ingeniería Química y Metalurgia de la Universidad Nacional de San Cristóbal de Huamanga. La investigación consistió en el modelamiento del CAD SolidWorks v.2018 con la finalidad de realizar los distintos despieces y analizar las estructuras 
móviles y estáticas (simulación por elementos finitos), según el requerimiento de diseño, luego se desarrolló un Pack de programas de Microsoft Office en Word y Excel; donde, con el programa Word se redacta la memoria del análisis y con el Excel se manejan los datos de entrada y salida de momentos y cortantes; para este análisis se emplea el software Solidworks premium v. 2018 (Licencia gratuita para principiantes). Además, posteriormente se usó para simular, la ingeniería Asistida por Computadora (CAE) para reducir el riesgo en la toma de decisiones tempranas y amplía el campo de exploración de soluciones posibles facilitando los procesos de optimización.

Posteriormente, el prototipo se sumó en el Diseño para la Manufactura y Ensamble (DFMA) que analiza y cuantifica los diseños propuestos desde el punto de vista de la manufactura y del ensamble, el manejo de datos sobre características de fabricación y parámetros de montaje, análisis no solo de componente individual sino del conjunto del sistema y tolerancias admisibles. Esta herramienta facilita la comunicación entre diseño y fabricación o cualquier otra disciplina que forme parte en la determinación del costo final de un producto, este procedimiento se complementó con el Análisis de Modo y Efectos de Falla (AMEF) para evaluar los modos de falla y las causas asociadas con el diseño y procesos de manufactura de un nuevo producto; finalmente, se ejecuta la simulación para estimar sobre prototipos virtuales, el comportamiento físico futuro de producto, facilitando la detección rápida de fallas y las modificaciones respectivas al mismo.

La característica esencial del diseño, es que se aplicó el principio de máquinas simples y compuestas; como: principio de la palanca, el principio de torno (movimiento de rotación), el principio de la rueda (rodajes que transmiten movimiento y dirección de rotación, sin variador de velocidad). En su elemento estructural destaca un cuerpo estático (Estructura o base) que soporta el esfuerzo cortante, flexión y fatiga (movimiento y la vibración) del cuerpo móvil (eje de paletas de cuchillas), que es accionada por un motor eléctrico de baja rpm (revoluciones por minuto), y que responde al principio de cizallamiento de cuerpos, donde la abertura de alimentación de la materia prima es de plataforma abierta y es ajustable a un mínimo de holgura para desfibrado de 0,5; 1 y $1,3 \mathrm{~mm}$, lo que le hace "preciso" en el momento de procesar la corteza de piña golden, constituido por elementos desarmables o desmontables, elementos de transmisión y fijación; todo el proceso es en batch. Incluye al sistema un mecanismo de descarga de desperdicios del proceso.

\section{Figura 1}

Características del diseño del prototipo en 3D. Vista lateral del rotor con paletas

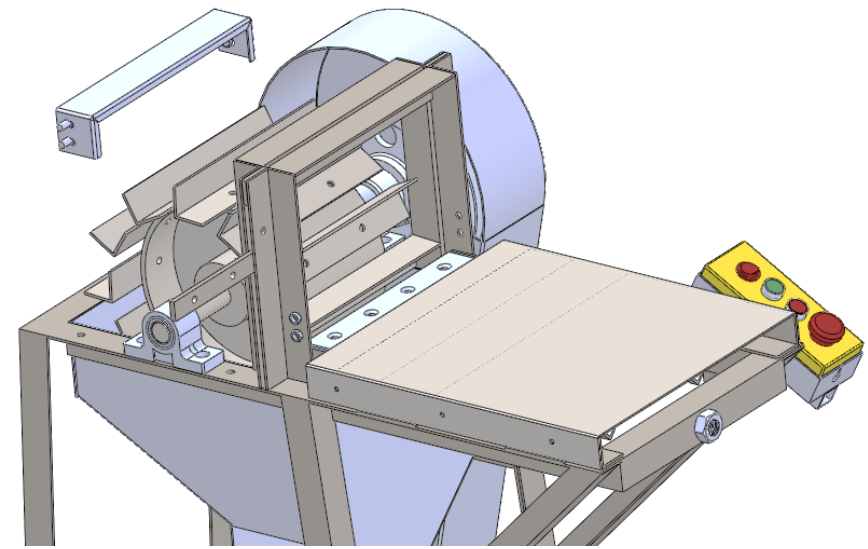

Nota. Elaboración propia. 
Posteriormente, con la maquina concluida en su construcción se procede a la prueba de operación, en vacío y con la materia prima, pero acondicionada con anticipación (condición de operación) y así obtener el adecuado rendimiento del sistema de desfibrado.

Las diferentes etapas para el diseño, son actividades relacionadas entre sí, que según el requerimiento de diseño, elaboración de croquis, selección del diseño en forma, elaboración de planos en 3D y 2D, ensamblado de partes y de simulación; hasta la validación del diseño en la puesta en marcha sin i con carga, se muestran en la figura 2.

\section{Figura 2}

Etapa de construcción de la máquina (Secuencial, ordenado y retroalimentable)

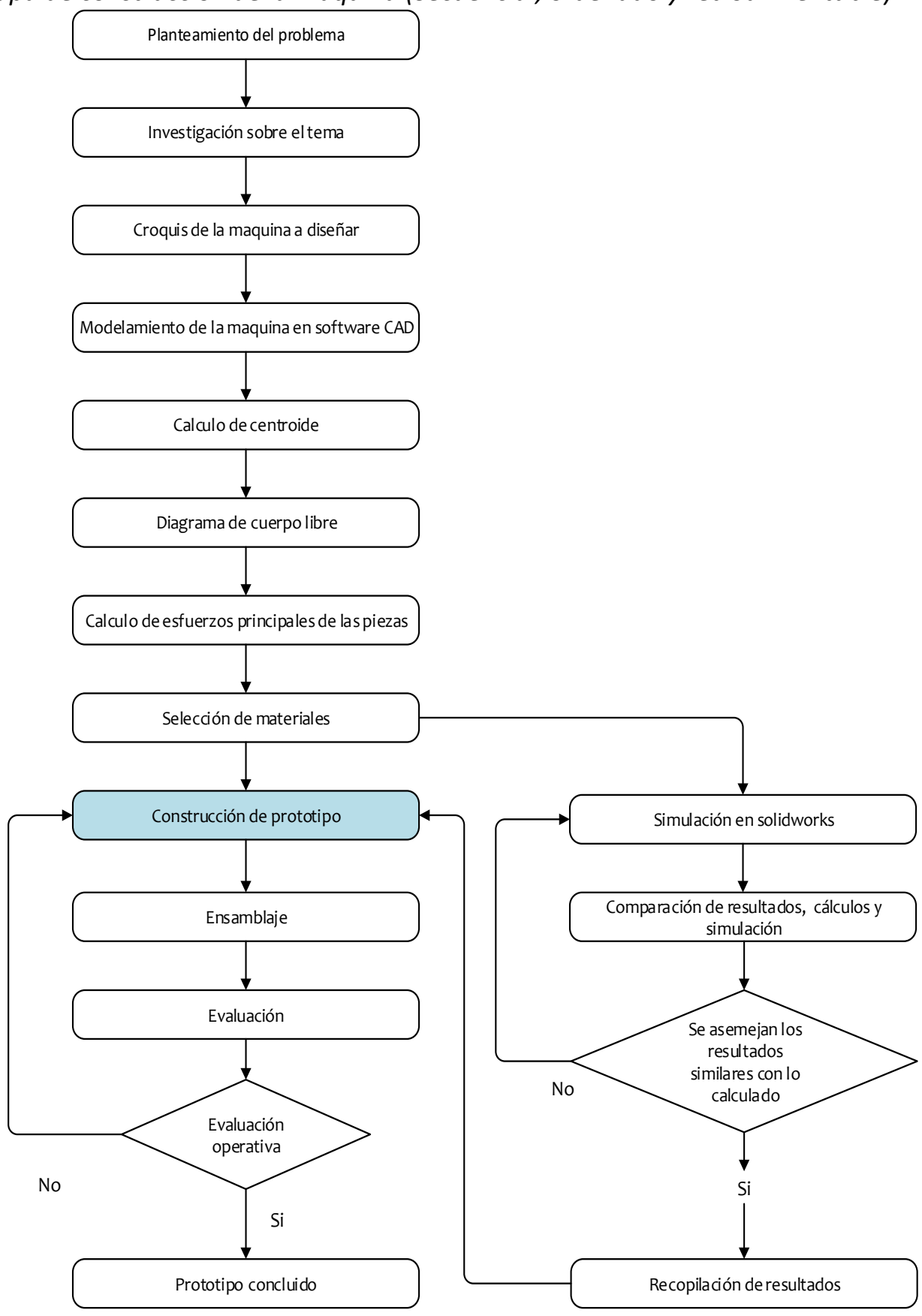

Nota. Elaboración propia. 


\section{Construcción de prototipo}

En la figura 3, se observan los siguientes pasos para la materialización del diseño en función de la manufactura de las diversas partes o piezas, con la aplicación de las máquinas-herramientas y herramientas manuales. Para ello, anticipadamente, realizamos los trazos según las medidas, corte, plegado y conformado de cada pieza.

\section{Figura 3}

Construcción de máquina (Herramientas auxiliares/despieces por piezas/despieces por componentes del prototipo)

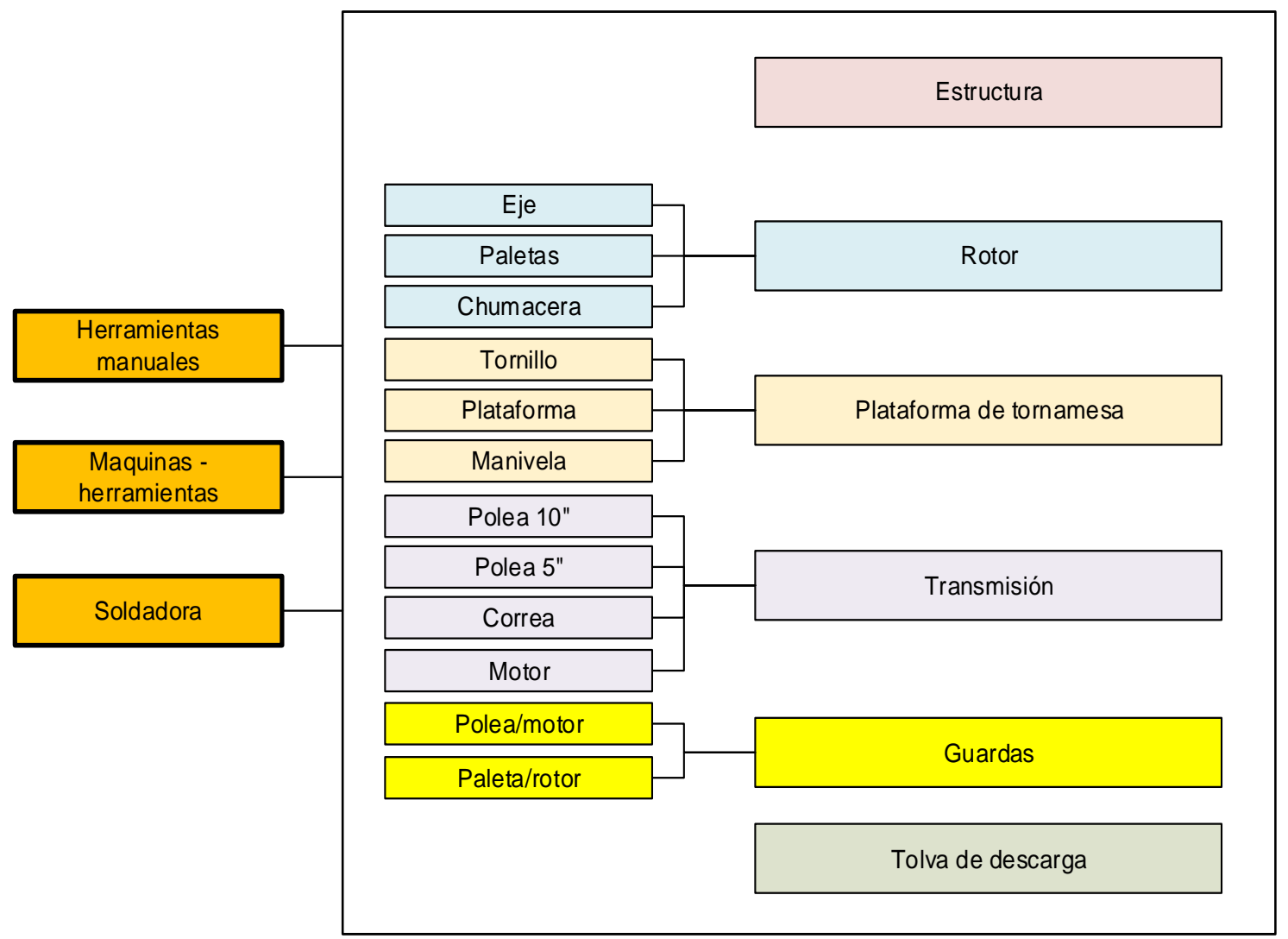

Nota. Elaboración propia.

\section{Ensamblaje}

Lo siguiente es el armado o "ensamble", según los "bosquejos" preliminares, que se aprecia en la figura 4, el procedimiento consiste en unir las piezas según la secuencia lógica de su funcionamiento, siguiendo ciertos protocolos de seguridad del ensamblador. 


\section{Figura 4}

Secuencia de ensamblado (Despieces por pieza/despieces por componentes/ensamblado final)

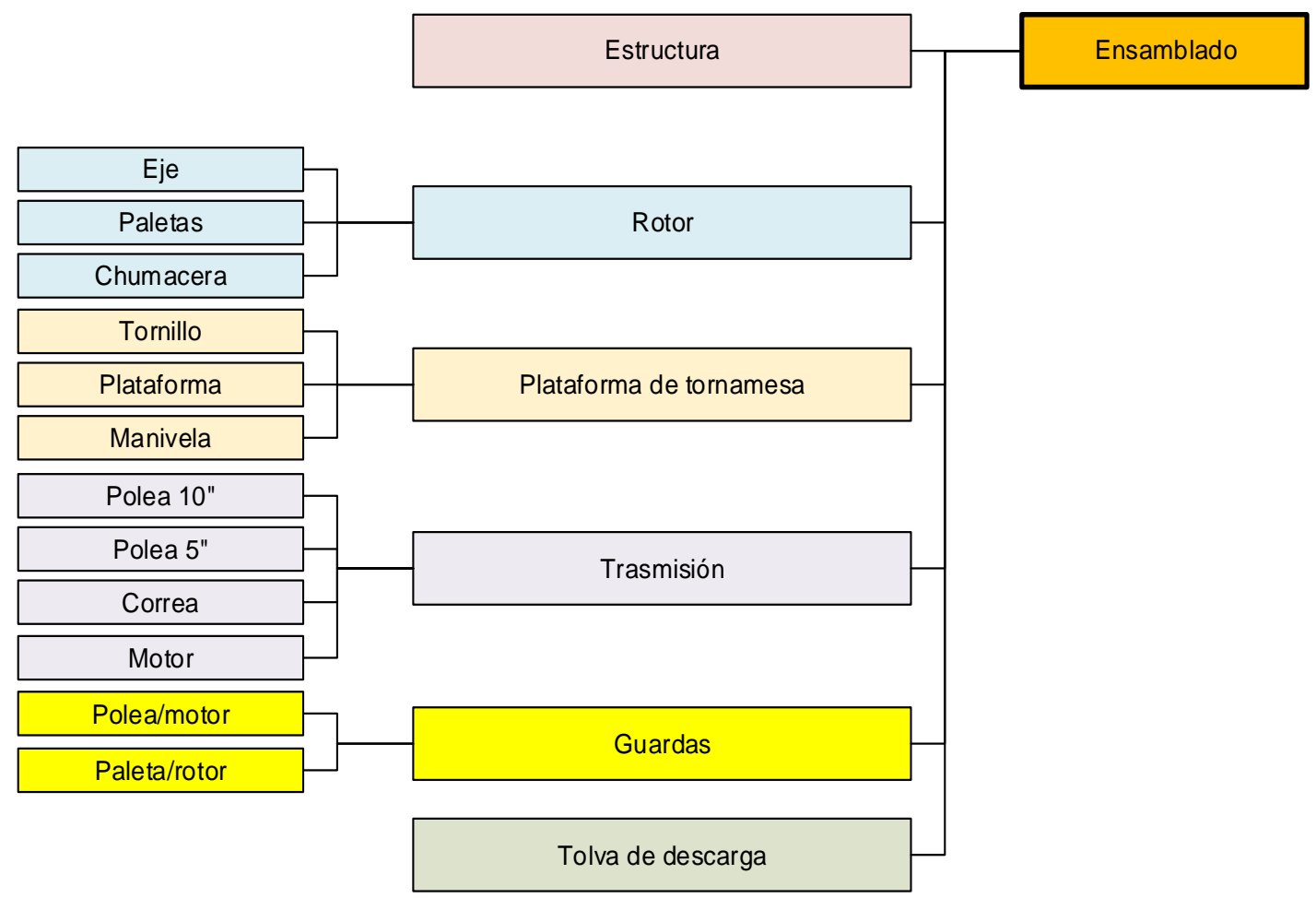

Nota. Elaboración propia.

\section{Evaluación operativa}

En etapa se realizó el control de la precisión de los mecanismos, que actúan en el desfibrado de la materia prima, al respecto de otros factores como la vibración, flexión, tensión y fatiga, y así de la potencia aplicada en la transmisión. Entonces, el control es primeramente visual y luego auditiva.

\section{Prototipo concluido}

Una vez terminada en su integridad, la maquina es un mecanismo tecnológico; que se evaluó y corrigió funcionalmente, para que actúe satisfactoriamente en la operación del desfibrado de los residuos de piña, el prototipo concluido se muestra en la figura 5. 
Figura 5

Prototipo concluido

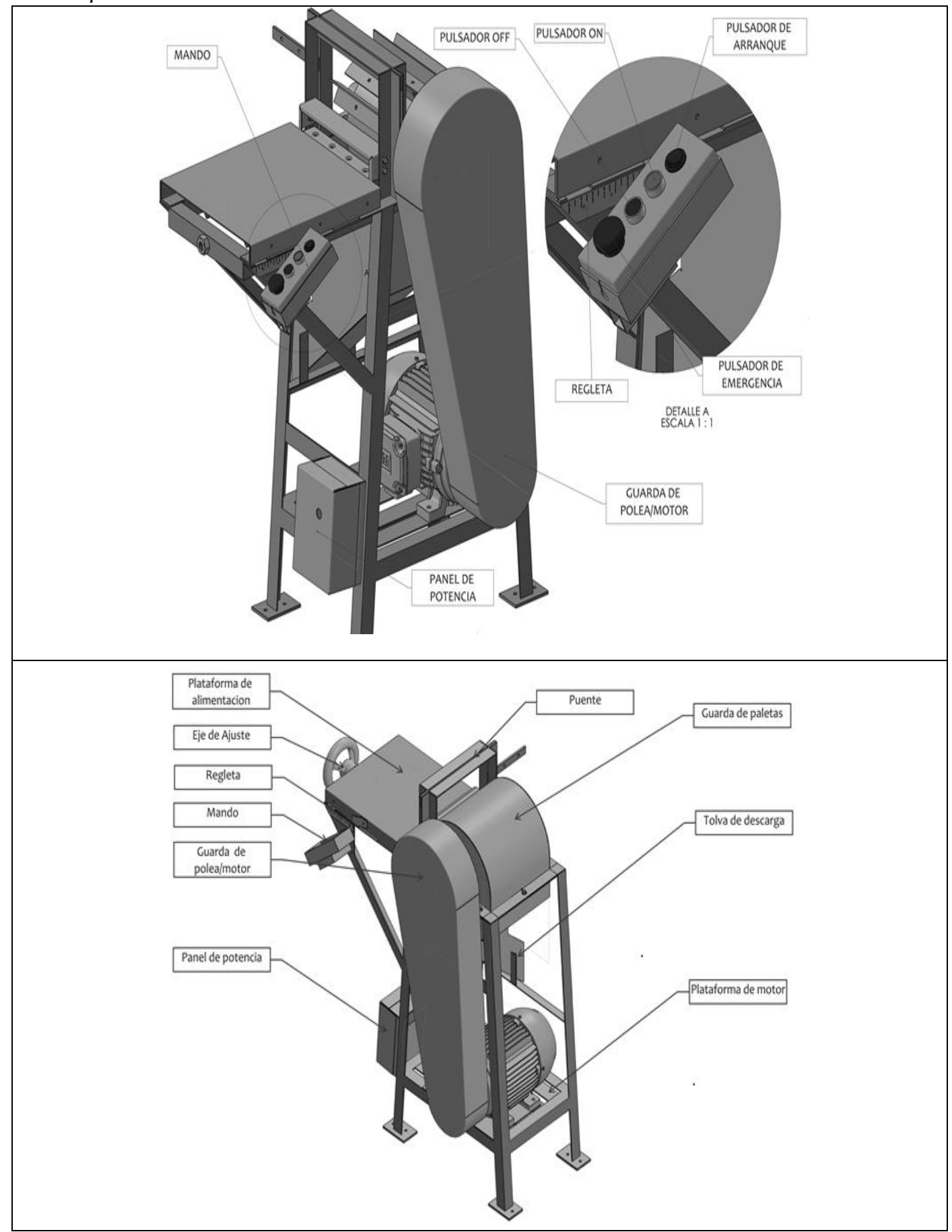

Nota. Elaboración propia.

\section{Resultados}

La característica esencial del diseño, es que maquina motriz accionado por un motor de baja potencia y torque, es exclusivo para cortezas blandas de poca resistencia mecánica como la piña, es precisa ya que desfibra con una holgura entre cuchillas, es desarmable y todo el proceso es en batch, 
de cada proceso realizado antes/durante/después del funcionamiento se evalúa, es netamente de acero inoxidable (paletas/tambor) con estructura de acero estructural. Segmentándose en 2 cuerpos estructurales específicos como el: cuerpo estático (estructura) que soporta el esfuerzo, (movimiento y la vibración) del cuerpo móvil (eje de paletas), que es accionada por un motor de baja rpm (revoluciones por minuto), y que responde al principio de cizallamiento de cuerpos, o comprensión inducido por una superficie metálico, donde la abertura de alimentación de la materia prima es ajustable a un mínimo de holgura para desfibrado de 0,5; 1 y 1,3 $\mathrm{mm}$ (según la necesidad del desfibrado).

\section{Prueba de operación sin carga}

Prueba visual y auditiva

Primeramente, estas pruebas realizadas después de la culminación de su construcción y ensamblado de la máquina, se ajusta a ciertos protocolos de seguridad para los usuarios y el cuidado para maquina en sí; correspondiente al examen minucioso de todas las piezas en forma visual (acabado de soldadura, partes desmontables, cableado eléctrico) y auditiva (sonido, de las paletas en su centro axial de giro para consta ningún sonido anormal al girar las paletas).

\section{Prueba de operación con carga}

\section{Evaluación de la holgura del desfibrado}

En etapa el propósito de esta prueba consiste en verificar si cumple con los criterios, condiciones de operación y las especificaciones técnicas del diseño para la amplitud de trabajo a realizar.

La holgura de desfibrado consiste en la abertura ajustable $(0,5$ a $100 \mathrm{~mm})$ de la cuchilla y contracuchilla que se requiere para introducir la corteza de la piña a fin de separar la fibra de la corteza, consta de paletas móviles radialmente sobre una paleta fija empotrada en la estructura; entonces, la regulación de la holgura de desfibrado se realizó con un calibre de espesores el cual se colocó entre la cuchilla y la contracuchilla. La holgura de desfibrado se varia en ciertos rangos diferentes, siendo $\mathrm{Df}=0,5 ; 1,0$ y $1,3 \mathrm{~mm}$. La regulación se hace con ayuda de un calibre de espesores.

En primer lugar, se delimito en la hoja la distancia a desfibrar, que en este caso fue de 0.4 a $0.5 \mathrm{~m}$ a partir del estrechamiento de la sección transversal, al excedente de hoja se le realiza un corte. A esta distancia se le adiciona una distancia adicional para el agarre, pues por motivos de seguridad no se efectúa el desfibrado de esta parte. Posteriormente la hoja fue divida a la mitad en la dirección de las fibras. Esta división se realiza con el propósito de evitar la forma cóncava de la hoja en su sección transversal, pues dada la rigidez de la hoja de la piña no quede asentada en la contracuchilla y dificulta el desfibrado. Finalmente, estas porciones de las hojas fueron pesadas antes del desfibrado.

\section{Evaluación de desfibrado de residuos de piña}

El espesor y largo promedio de las hojas de piña (variedad Golden) ha seleccionar, con esta evaluación, se plantea por consiguiente la apertura necesaria entre las paletas del rotor y la cuchilla 
fija de la mesa (plataforma) móvil del desfibrador (figura 6), las pruebas de aperturas mencionadas anteriormente está en función al rendimiento a obtener (a mayor cantidad de fibra limpia obtenido, mayor rendimiento de desfibrado).

Espín (2015), refiere que existe una gran variedad de métodos de desfibrado de acuerdo con el tipo de hojas o pseudotallos y a las características propias de cada especie. Entre algunas especies existen grandes diferencias de espesores, cantidad de líquido, resistencia y flexibilidad por lo que un método puede ser más adecuado que otro al momento de la operación.

\section{Figura 6}

Residuos de bagazo en función a diferentes aperturas entre cuchillas ( $\mathrm{mm}$ ).

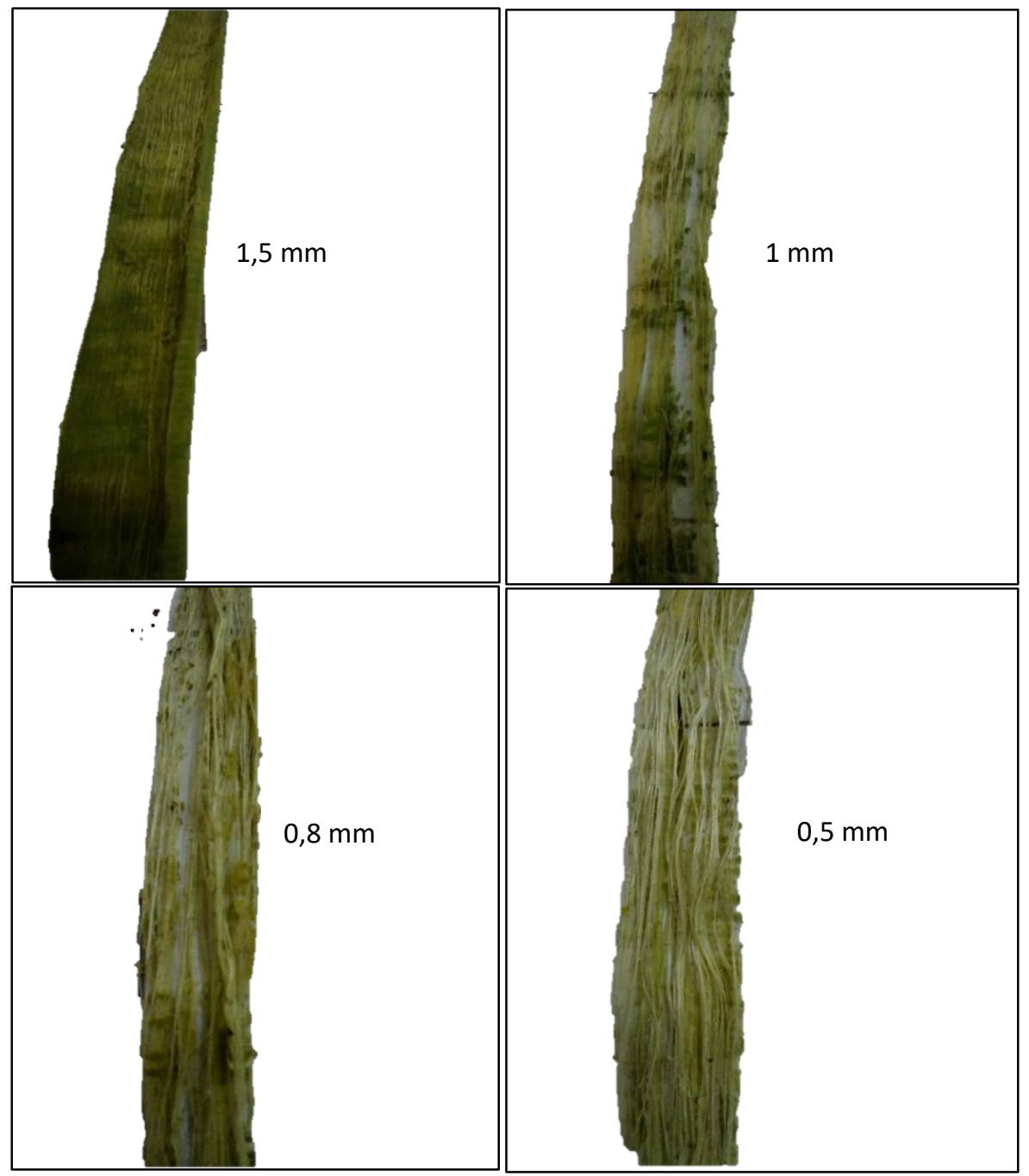

Nota. Elaboración propia.

\section{Discusión}

Las normas establecidas para la etapa de diseño, construcción y evaluación se centraron en la realización de las restricciones y estándares de diseño, normado por la ISO (International Standarization Organization), tanto como UNE-EN ISO 5457 (2000), que establece varios aspectos técnicos para la elaboración de un formato, de los cuales se elaboran los planos preliminares para la construcción, UNE-EN ISO 13850: 2008. Seguridad de las máquinas, Parada de emergencia. 
Principios para el diseño, (ISO 13850:2006), UNE-EN ISO 14738:2003/AC:2005. Seguridad de las máquinas. Requisitos antropométricos para el diseño de puestos de trabajo asociados a máquinas, UNE-EN 614-2:2001+A1:2008. Evaluación de las posturas y movimientos de trabajo en relación con las máquinas, UNE-EN 894-3:2001+A1:2009. Seguridad de las máquinas. Requisitos ergonómicos para el diseño de dispositivos de información y mandos.

La evaluación requerida se encamino por los procedimientos normales de seguridad de cualquier equipo o máquina, y como materia prima se llegó a la conclusión de que debe ser de corteza húmeda, es decir cosechada en el momento de su máxima longitud, para obtener fibras más homogéneas y prever posibles rupturas y/o corte de las fibras.

\section{Conclusiones}

Con el sistema de desfibrado en este proyecto se constata que los parámetros de diseño, son: 1) los materiales de construcción deben ser mixtas, es decir con acero estructural (no menor de $2 \mathrm{~mm}$, y no mayor de $6 \mathrm{~mm}$; de espesor), galvanizado ( $2 \mathrm{~mm}$, en espesor) e inoxidable ( $2 \mathrm{~mm}$, en espesor), 2) y el sistema de sujeción, en la mayoría debe ser por soldadura eléctrica y TIG (Tungsten Inert Gas), con adición de aporte, 3) la construcción, se realiza de la estructura principal a las demás partes secundarias, 4) la calibración de cuchilla y la contracuchilla para el proceso de desfibrado, es según el avance de giro del tornillo (de la tornamesa), lo que da un rango de barrido máximo de 20 $\mathrm{mm}$, ya que el requerimiento de la abertura (de la alimentación) es de 0,5 a 1,3 mm.

Los criterios funcionales se ajustan a las dimensiones de la máquina y la materia prima, debido a que el proceso radica en el proceso de cizallamiento de la corteza, con una parte estática (contracuchilla) y otra móvil (cuchilla); en este sentido se requiere un grado de precisión para una adecuada desfibrada.

Los criterios técnicos radican en la constante calibrada de la cuchilla y la contracuchilla, ya que, por cada operación con la materia prima, es necesario supervisar cada componente móvil. El manejo, control y mantenimiento; son los parámetros es crítico en el momento de funcionamiento de la máquina, porque es una máquina de precisión, y como es de operación peligrosa el control es en base de un sistema de emergencia, que permita tener el control inmediato sobre la máquina. Para la adecuada operación se requiere de varios "breaker" de funcionamiento independiente de cada tiempo promedio. En el mantenimiento es periódico, es decir de cada 2 horas de funcionamiento, se necesita un mantenimiento predictivo, preventivo y correctivo.

Para evitar que las partes móviles de la máquina tengan problemas por acumulación de depósitos de corteza, es recomendable realizar la limpieza constante en cada operación, esto por seguridad industrial.

La energía consumida en el proceso de desfibrado durante la entrada de la hoja al órgano desfibrador tiene una dependencia bien marcada respecto a la velocidad de alimentación, pues en la medida que aumenta la velocidad de alimentación disminuye exponencialmente la energía consumida, lo que no se faculte en el cambio sustancial de la operación, siempre se debe evaluar el manejo y el control. 


\section{Referencias Bibliográficas}

Avella C., Valenzuela A., Gonzales M., Gomez., Gamboni S., (2018). Aprovechamiento residuos biomasa de producción de piña (Ananas comosus) en el municipio de Aguazul Casare. Colombia. Extraído (PDF): www.academia.edu.

Blanco, M. (2018). Tesis doctoral. Metodología de diseño de máquinas apropiadas para contextos de comunidades en desarrollo. Universitat Politécnica de Catalunya. España.

Budynas, R. y Nisbett, K. (2008). Diseño en ingeniería mecánica de Shigley. 8 va. Edición. Edit. McGraw-Hill. México D.F.

Deutschman A. D. (1987). Diseño de máquinas. Teoría y prácticas. Edit. Continental. México.

Espín, L. y Tello, J. (2015). Diseño y construcción de una desfibradora de hojas y pseudotallos para obtener material lignocelulósico a utilizar como refuerzo de polímeros. Escuela Politécnica Nacional. Quito. Ecuador.

Faires V.M. (199...). Diseño de elementos de máquinas. Edit. Montaner y Simón S.A. Barcelona. España.

Gómez R. (2006). Normalización técnica aplicada a la ingeniería. Lima. Perú.

Hibbeler, R. C. (2006). Mecánica de materiales. 6o Edición. Editorial Pearson Prentice Hall. México.

Niemann G. (1973). Tratado teórico-práctico de elementos de máquina. Cálculo, diseño y construcción. Edit. Labor S.A. Alemania.

Pérez, R. el at (2013). Diseño y construcción de una desfibradora de hojas de Agave angustifolia Haw. Revista Ciencias Técnicas Agropecuarias. Vol. 22. Núm. 4. pp 5-14. México.

Pope, J. E. (2000). Soluciones prácticas para el ingeniero mecánico. Editorial McGrawHill. México.

Rosell, D. el at (2003). Teoría funcional de una máquina desfibradora de Lechugilla (Agave lechuguilla Torr) de tipo tambor. Naturaleza y desarrollo. Vol. 1. Núm. 2. México.

Villegas M. et al (2013). Fibras textiles naturales sustentables y nuevos hábitos de consumo. Revista legada de arquitectura y diseño. № 13. Pp. 31-45. Universidad Autónoma del Estado de México. México.

Zapata J. (2013). Diseño de elementos de máquinas I. 1o edición. Piura. Perú.

Rondón M. O. El diseño de la ingeniería de máquina y su evolución histórica, versión PDF, consultado en http://casanchi.com/ref/ingemaquina01.pdf

Diseño asistido por computadora, consultado en: http://es.scribd.com/doc/27237244/Libro-deDiseNode-mAquinas\#scribd

Introducción a las maquinas simples y compuestas, consultado en: http://www.salonhogar.net/Salones/Ciencias/13/Maquinas_simples_compuestas/ Simples_compuestas.htm

Mecanismos de máquinas, consultado en:

http://concurso.cnice.mec.es/cnice2006/material107/maquinas/maq_compuesta. htm. 